- Basic Research

\title{
Effect of Perceived Devaluation, Discrimination, and Internalized Stigma on Adherence to Medication among Patients with Mental Illness.
}

\author{
Zienab Mohammed Ibrahim Morsi *, Fatma Mohammed Ibrahim Morsi** \\ *Lecturer of Psychiatric/Mental Health Nursing -Faculty of Nursing -MTI \\ **Lecturer of Psychiatric/Mental Health Nursing- Faculty of Nursing - Ain Shams University
}

\begin{abstract}
Introduction: Perceived and internalized stigma has significant impact on many aspects of life among patients with mental ill. Nonadherence to treatment increases the readmission rates, leads to poor prognosis of illness and worsening the symptoms. Aim: This study was conducted to determine the Effect of Perceived Devaluation, Discrimination, and Internalized Stigma on Adherence to Medication among Patients with Mental Illness. Methods: Descriptive correlational design was utilized in the current study on a sample of 189 patients with mental illness from psychiatric outpatient clinics at Abassia. Mental Health Hospital. Tools: data was collected using: 1- A structured interview schedule to assess demographic and clinical "psychiatric" data, 2Perceived Devaluation/Discrimination Scale, 3- Internalized Stigma of Mental Illness Questionnaire, and 4- Drug Attitude Inventory. Results: data analysis revealed that, two thirds of the studied patients had high level (midpoint above 2.5) of perceived devaluation/discrimination (societal) stigma, three quarters of them experienced internalized (self) stigma of mental illness, and less than three fifths of them had negative attitude toward psychotropic medications. There were highly statistically indirect significant relations between patient attitude towards psychotropic medications and their levels of internalized (self) stigma, and perceived devaluation/discrimination (societal stigma). Also, there was a statistically direct significant difference between internalized (self) stigma and perceived devaluation/discrimination (societal stigma). Conclusion: The levels of societal stigma and internalized (self) stigma was determined to be high and negatively affected the patients' attitudes toward psychotropic medications and adherence. Recommendation: Supportive and rehabilitative counseling interventions are required for patients with mental illness and their families to improve their stigma resistance, reduce their self-stigma and enhance drug adherence.
\end{abstract}

Keywords: Devaluation, Discrimination, Internalized Stigma, Adherence, Medication, Mental Illness 


\section{Introduction}

Stigma is a societal phenomenon in which the general public regards people with certain characteristics as being inferior, dangerous, or having other negative attributes. The most serious correlates of stigma include mental diseases. Internalized stigma, also known as self-stigma, is a progressive process in which a person adopts unfavorable societal preconceptions regarding traits that are ridiculed by others (Corrigan, Rafacz and Rüsch, 2011).

Stigma of mental illness can be categorized into societal stigma and selfstigma. societal stigma is the prejudice endorsed by the general population and manifests as discrimination toward individuals with mental illness. Self-stigma refers to patients' beliefs about prevalent negative stereotypes and attitudes of general population toward their condition and toward themselves as potentially stigmatized group or from the actual experience of discrimination (Lee, 2016; Boyd, Adler, Otilingam, \& Peters, 2014; and Corrigan and Rao, 2012). Internalized stigma involves cognitive (Self-defeating thoughts, sense of inferiority, sense of incompetence, negative sense of self etc.), affective (feelings of despondency, sadness, embarrassment, shame, anger etc.) and behavioral (selfdenigration, self-isolation, concealment of status, social withdrawal, social avoidance etc.) responses to perceived or experienced stigma (Sarısoy, Kaçar, Pazvantoğlu, Korkmaz, Öztürk, Akkaya, \& Sahin, 2013).

Stigma and discrimination experienced by people with mental illness led to depression, low self-esteem, impaired quality of life, poor recovery orientation, poor symptomatic outcome and block certain life goals such as living independently, stable employment and meaningful education (Keen, George, Scragg, \& Peters, 2017; and Zäske, Degner, Jockers-Scherübl, Klingberg, Klosterkötter, Maier, \& Gaebel, 2016). Furthermore, people often avoid the use of services for mental health because of fear of being labeled as a" mentally ill patient" or discriminated (Teferra, Hanlon, Beyero, Jacobsson, and Shibre, 2013).

Patients are usually aware of stereotypes that society holds about people receiving psychiatric care and are concerned about them. Anxiety about potential stigma increases in those who have a greater tendency toward self-stigma. The consequences may be even more serious than in the case of stigmatization by others (Demoz, Legesse, Teklay, Demeke, Eyob, Shewamene, and Abera 2014).

In addition, adherence to treatment plan can be interrupted and $\underline{\text { discontinuation of medication can result from self-stigma leading to more }}$ 
deterioration in social, psychological, occupational, and academic life. Patients with poor treatment adherence leads to increased hospitalization, higher healthcare costs and is a predictor of poor outcomes like more relapses, suicide, and overall mortality (Feldhaus, Falke, von Gruchalla, Maisch, Uhlmann, Bock, \& Lencer, 2018; and Jenkins, and Carpenter-Song, 2009).

Non-adherence to psychiatric treatment regimens has a profound impact on the disease course, relapse, future recovery, cost of health care, and the outcome for the patient. Fear of stigma connected to the diagnosis and fear of rejection due to revealing symptoms have been suggested to further increase non-adherence (Keen, et al., 2017; and Sorsdahl, Kakuma, Wilson, and Stein, 2012).

Little is known about the internalized stigma experienced by patients with mental illness, nor is there a clear understanding of the relationship between internalized stigma, empowerment, and perceived devaluation and discrimination among mental ill patients (Tanabe, Hayashi, and Ideno, 2016).

\section{Significance of the Study:}

Many of the negative stereotypes and myths about mental illness are framed around individuals with serious mental illness, and those individuals will likely suffer the greatest disease burden without treatment. However, the impact of stigma can be felt across the spectrum of mental disorders, conditions, and levels of impairment. As a barrier to treatment, anticipated discrimination and stigma is impactful among individuals who are experiencing less severe mental illness or psychiatric impairment (Quinn, Williams, \& Weisz, 2015).

Despite the critical importance of medication, non-adherence to the prescribed drug treatments has been recognized as a worldwide problem and may be the most challenging aspect of treating patients with mental illness (Yılmaz, \& Okanl, 2015).

The Egyptian literature has very few studies that explore the relation between type of stigma and find out which one is more influential Gaber $\boldsymbol{\&}$ Wadie, (2016). Furthermore, nurses should recognize the detrimental impact of stigmatization on the psychological well-being of patients to be able to provide patients with psychosocial care and support. The data obtained in the study will 
pave the way for further studies and bridge an important gap in the literature about the combination of various factors linked to societal and self-stigma, and treatment adherence.

\section{Aim of the Study:}

The aim of this study was to determine the effect of perceived devaluation, discrimination, and internalized stigma on adherence to medication among patients with mental illness.

\section{Research questions:}

1. What are the patients ' perception regarding societal attitude (devaluation and discrimination) toward mental illness?

2. Are patients with mental illness experiencing internalized stigma?

3. Do patients with mental illness adhere to medications?

4. What are the correlations among patients' perception regarding societal attitude, internalized stigma, and their adherence to medications?

Operational definition: In the present study societal attitude refers to devaluation and discrimination of the public toward patients with mental illness.

\section{Subjects and Method:}

Design: A descriptive correlational design was used to examine the effect of perceived devaluation, discrimination, and internalized stigma on adherence to medication among patients with mental illness.

Setting: This study was conducted at outpatient clinics at Abassia Mental Health Hospital, affiliated to Mental Health Secretariat,

Subjects: A purposive sample of 189 adult patients with mental illness according to certain inclusion criteria, who were available at the time of the study, and determined by using appropriate statistical equation.

$\mathrm{S}=\mathrm{X}^{2} \mathrm{NP}(1-\mathrm{P}) \div \mathrm{d}^{2}(\mathrm{~N}-1)+\mathrm{X}^{2} \mathrm{P}(1-\mathrm{P})$.

$\mathrm{s}=$ required sample size

$\mathrm{x}=$ the table value of chi-square for 1 degree of freedom at the desired confidence level (3.841)

$\mathrm{N}=$ the population size. 
$\mathrm{P}=$ the population proportion (assumed to be .50 since this would provide the maximum sample size)

$\mathrm{d}=$ the degree of accuracy expressed as a proportion (.05)

(Krejcie, \& Morgan, 1970).

\section{Inclusion criteria:}

- Adult patients.

- Both genders.

- All educational levels and different socioeconomic classes.

- Diagnosed with psychiatric illnesses for at least 1 year.

- Open for communication and cooperation.

- Insight with his/her psychiatric condition.

Exclusion criteria: Diagnoses of mental retardation, organic brain syndrome, epilepsy, substance use disorders are excluded from the study sample.

\section{Tools of data collection:}

I) A structured interview schedule: to assess patient's demographic characteristics and clinical "psychiatric" data: this includes age, gender, educational level, occupational status, adequacy of monthly income, medical diagnosis, type of treatments, duration of illness, and previous hospital admission.

\section{II) Perceived Devaluation - Discrimination Scale (PDD):}

This scale was developed by Link, Yang, Phelan, \& Collins (2004), is a 12-items tool which measures the extent to which a person believes that most people will devalue or discriminate against someone with a mental illness. PDD is measured on a 4-point Likert scale with possible scores ranging from 1 to 4 agreement scale $(1=$ strongly disagree, $2=$ disagree, $3=$ agree, and 4 $=$ strongly agree), so that a higher score indicates a higher level of perceived stigma. Items 1, 2, 3, 4, 8, and 10 are reverse scored. Scoring: all answers to be summed and divided by the number of items in the scale, the scores are dichotomized to midpoint of 2.5 on PDD scales. In this study, reliability (Pearson's r=0.84) and, the Cronbach's "coefficient of the PDD was .89.

Stigma perception

\begin{tabular}{|c|c|}
\hline Low & High \\
\hline$\leq 2.5$ & $>2.5$ \\
\hline
\end{tabular}




\section{III) Internalized Stigma of Mental Illness (ISMI) scale:}

(Ritsher, Otilingam, \& Grajales, 2003): The ISMI is a 29-item scale that assesses self-stigma. It is composed of 5 subscales: Alienation, Stereotype Endorsement, Perceived Discrimination, Social Withdrawal and Stigma Resistance. Each item is rated on a four-point Likert scale from 1 (strongly dis-agree) to 4 (strongly agree). The five stigma resistance subscale items are reverse-coded and serve as a validity check. The stigma resistance score is calculated by substracting the actual value from five. Therefore, stigma resistance displays the same direction of correlation as the other four subscales. A high total score on the ISMI scale indicates more severe internalized stigmatization. Strong internal consistency $(\alpha=0.90)$ and testretest reliability $(r=0.92)$ have been reported for the ISMI (Ritsher and Phelan, 2004). The score in each domain was calculated by adding the item scores together and then dividing them by the total number of answered items. The resulting score should range from 1-4. In the current study the 2-category method was used, the interpretations of the results were as follows according to Boyd, et al., (2014).:

\begin{tabular}{|l|c|c|}
\hline \multirow{2}{*}{ Domains } & \multicolumn{2}{c|}{ Scores } \\
& No/ minimal & Severe \\
\hline Alienation & & \\
\cline { 1 - 1 } Stereotype Endorsement & \multirow{2}{*}{$1: 2.5$} & \multirow{2}{*}{$2.6: 4$} \\
\hline Perceived Discrimination & \\
\cline { 1 - 1 } Social Withdrawal & & \\
\hline Stigma Resistance & & \\
\hline Internalized stigma & & \\
\hline
\end{tabular}

In this study, reliability (Pearson's $r=0.80$ ) and, the Cronbach's "coefficient of the ISMI was .91.

\section{IV) Drug Attitude Inventory (DAI)-10 (Awad, 1993):}

It includes a series of questions completed by the patient, each with true/false answers, pertaining to various aspects of the patient's perceptions and experiences of treatment. To calculate the score from a set of answers, each 'positive' answer is given a score of plus one, and each 'negative' answer is given a score of minus one. The DAI- 10 scoring ranges from -10 to +10 with a total score $>0$ indicating a positive attitude toward psychiatric medications and a total score of $<0$ indicating a negative attitude toward 
psychiatric medications. In this study, reliability (Pearson's $r=0.87$ ) and, the Cronbach's "coefficient of the DAI was .86.

Operational Design: The operational design for this study includes preparatory phase, pilot study, field work, limitations of the study, and ethical considerations.

Preparatory phase: It included reviewing theoretical knowledge of various aspects of societal stigma, internalized stigma of patient with mental illness, and adherence and discontinuation of psychotropic medication in past, recent, national, and international literature that are available in books, articles, internet, periodicals, and magazines.

The researchers prepared and designed tools of data collection. Then the tools were translated into Arabic language and back translated into English language by language experts, and any discrepancies elicited between the back translation and the original tools were taken as an indication of translation error.

Tool validity and reliability: Tool's validity was proved by experts in Psychiatric/Mental Health Nursing, Psychiatric Medicine and Community Health Nursing, their opinions were elicited as regards to the tool format layout, consistency, knowledge accuracy, relevance, and competencies as well the scoring system. Internal consistency (Cronbach alpha) and Pearson correlation coefficient (r) were tested for each tool.

\section{Pilot study:}

The pilot study was conducted on a ratio of $10 \%$ of the study sample size "19 patients" who were later excluded from the study subjects and substituted with other 19 patients who fulfilled the previously mentioned criteria, to evaluate the clarity, applicability, and feasibility of the research tools and to estimate the time needed for data collection. The tool was finalized based on the results of the pilot study.

\section{Field work:}

Data collection took seven months during the period from the beginning of June 2019 to the end of December 2019. Data were collected through 2 days/week during morning shift (9.00 a.m.: 12.00 p.m.) for 20-30 minutes for each case. The researchers met each patient individually and explained the purpose and nature of the study, then the questionnaire form was distributed to be completed by selecting only one response that reflects the actual situation. The researchers 
asked the patients about any difficulties that faced them during answering the questionnaires and offered help.

\section{Administrative Design}

An official letter was issued from the Faculty of Nursing-MTI to the director of Abasia Mental Health Hospital, explaining the aim of the study and requesting their permission for data collection. Oral consent was obtained from every patient who was included in the study.

\section{Ethical considerations}

Participation was voluntary and anonymity was assured. Consent was taken from each participant. The participants were informed about their right to withdraw from the study at any time. Subjects were assured about confidentiality of the information gathered and its use only for their benefits and for the purpose of the study.

\section{Statistical Design}

In the present study, the statistical analysis of the data was done by using computer software for excel program and statistical package for social science SPSS version 20.0. Data were presented using descriptive statistics in the form of frequencies and percentages for categorical data. The statistical significance and association were assessed using Qui square test and Pearson correlation coefficient. The observed differences, associations were considered as follows: $\mathrm{P}$ $>0.05$ not significant $(\mathrm{NS}) ; \mathrm{P}<0.05$ significant $(\mathrm{S})$; and $\mathrm{P}<0.001$ high significant (HS).

\section{Results}

Table (1) reveals the distribution of the studied patients according to their demographic characteristics. According to their gender, 57.1\% of them were male, $51.9 \%$ of them were in the age group of $28 \leq 38$ years old, and their mean age was $31.7 \pm 9.6$ years old, also, $64.6 \%$ of them lived in rural areas. Regarding their educational level, $37 \%$ of the studied patients had finished primary education, $67.3 \%$ of them were unemployed, and $59.8 \%$ had an inadequate monthly income.

Table (2) shows the distribution of the studied patients according to their clinical "psychiatric" data. Regarding their psychiatric diagnosis, $48.2 \%$ of them 
were diagnosed with schizophrenia, and $42.3 \%$ of them were diagnosed with mood disorders. As regards the duration of illness, $57.2 \%$ of them had a duration of illness of 3 to less than 6 years, all of them received pharmacological treatment, while only $21.6 \%$ of them received psychotherapy. Regarding disease relapse, $92.6 \%$ of the studied patients had previous hospitalization, and $37.1 \%$ of those patients were hospitalized for one time.

Table (3) shows that, the studied patients perceived high devaluation and discrimination from society as their mean score was 3.01, regarding internalized (self) stigma, they experienced severe internalized stigma, as their mean score was 2.59 , also they experienced severe alienation, severe stereotype endorsement, severe discrimination experience, severe social withdrawal, and no/minimal stigma resistance in which their mean scores were $2.75,2.04,3.16,2.7$, and 1.26 respectively. Regarding patients`attitude toward psychotropic medication, they experienced negative attitude as their mean score was 2.83 .

Regarding the distribution of the studied patients according to their levels of perceived devaluation/discrimination (societal stigma), Figure (1): illustrates that, $62.9 \%$ of them experienced high perceived devaluation/discrimination (societal stigma). Regarding the distribution of the studied patients according to their levels of internalized stigma, Figure (2): reveals that, 75.7\% experienced severe internalized stigma, while $24.3 \%$ of them experienced no/minimal internalized stigma.

Figure (3): illustrates the distribution of the studied patients according to their attitude toward psychotropic medications, and showed that $58.2 \%$ of them had negative attitude toward psychotropic medications, while $41.8 \%$ of them had positive attitude toward psychotropic medications

Regarding the relation between demographic characteristics of the studied patients and the study variables (their perceived devaluation/discrimination, internalized stigma of mental illness, and drug attitude),

Table (4): shows that, there were statistically significant differences between gender of the studied patients and levels of internalized stigma of mental illness, and patient attitude toward psychotropic medication in which $\mathrm{x}^{2}=21.7$ and 5.26 at $\mathrm{p}<0.001$, and $<0.05$ respectively. Also, there were statistically significant differences between perceived devaluation/discrimination of the studied patients and their occupational status, and residence in which $\mathrm{x}^{2}=85.6$ and 102.2 at $\mathrm{p}<0.001$. There were statistically significant differences between 
internalized stigma of mental illness of the studied patients and their occupational status, residence, and adequacy of monthly income, in which $\mathrm{x}^{2}=22.4,34.9$, and 18.6 at $\mathrm{p}<0.001$. Also, there were statistically significant differences between patients' attitude toward psychotropic medication and their occupational status, residence, and adequacy of monthly income, in which $\mathrm{x}^{2}=108.0,137.2,12.53$ at $\mathrm{p}<0.001$.

Table (5) shows that, there were statistically significant differences between psychiatric diagnosis of the studied patients and their perceived devaluation/discrimination, internalized stigma of mental illness, and attitude toward psychotropic medication in which $\mathrm{x}^{2}=58.8,62.0$ and 82.7 at $\mathrm{p}<0.001$. Also, there were statistically significant differences between perceived devaluation/discrimination of the studied patients and their occupational status, and residence in which $\mathrm{x}^{2}=85.6$ and 102.2 at $\mathrm{p}<0.001$. There were statistically significant differences between internalized stigma of mental illness of the studied patients and their occupational status, residence, and adequacy of monthly income, in which $\mathrm{x}^{2}=22.4,34.9$, and 18.6 at $\mathrm{p}<0.001$. Also, there were statistically significant differences between previous hospitalization of the studied patients and their perceived devaluation/discrimination, internalized stigma of mental illness, and attitude toward psychotropic medication in which $\mathrm{x}^{2}=25.7,8.83$ and 21.05 at $\mathrm{p}<0.001$. Also, there was a statistically significant difference between duration of illness of the studied patients and their attitude toward psychotropic medication in which $\mathrm{x}^{2}=73.3$ at $\mathrm{p}<0.001$.

Table (6): shows that, there were highly statistically indirect significant relations between patient attitude towards psychotropic medications and their levels of internalized stigma, and perceived devaluation/discrimination, in which $\mathrm{r}=-.669$, and -.905 at $\mathrm{p}<0.001$. In other words, patients who had severe internalized (self) stigma and high level of perceived devaluation/discrimination (societal) stigma, experienced negative attitude (not adhered) toward psychotropic medication. Also, there was a statistically direct significant difference between internalized (self) stigma and perceived devaluation/discrimination (societal) stigma, in which $\mathrm{r}=.739$ at $\mathrm{p}<0.001$. 
Table 1: Distribution of the studied patients according to their demographic characteristics $(n=189)$.

\begin{tabular}{|c|c|c|}
\hline Statement & No & $\%$ \\
\hline $\begin{array}{l}\text { Gender } \\
\text { - Male } \\
\text { - Female }\end{array}$ & $\begin{array}{c}108 \\
81\end{array}$ & $\begin{array}{l}57.1 \\
42.9\end{array}$ \\
\hline $\begin{array}{l}\text { Age } \\
\text { - } 18 \leq 28 \\
\text { - } 28 \leq 38 \\
\text { - } 38 \leq 48 \\
\text { - More than } 48\end{array}$ & $\begin{array}{c}68 \\
98 \\
15 \\
8 \\
\end{array}$ & $\begin{array}{c}35.9 \\
51.9 \\
7.9 \\
4.3 \\
\end{array}$ \\
\hline Mean \pm SD & \multicolumn{2}{|c|}{$31.7 \pm 9.6$} \\
\hline $\begin{array}{l}\text { Educational Status } \\
\text { - Illiterate } \\
\text { - Primary education } \\
\text { - Secondary education } \\
\text { - Diploma } \\
\text { - University education }\end{array}$ & $\begin{array}{r}29 \\
70 \\
28 \\
53 \\
9\end{array}$ & \begin{tabular}{c|c}
10.5 & \\
37 & 1 \\
14.8 \\
28.1 \\
4.8
\end{tabular} \\
\hline $\begin{array}{l}\text { Occupational Status } \\
\text { - Unemployed } \\
\text { - Private work } \\
\text { - Governmental work } \\
\text { - Retired }\end{array}$ & $\begin{array}{c}134 \\
38 \\
9 \\
8 \\
\end{array}$ & $\begin{array}{c}67.3 \\
32.7 \\
4.8 \\
4.2 \\
\end{array}$ \\
\hline $\begin{array}{l}\text { Residence } \\
\text { - Urban } \\
\text { - Rural }\end{array}$ & $\begin{array}{c}67 \\
122\end{array}$ & $\begin{array}{l}35.4 \\
64.6\end{array}$ \\
\hline Adequacy of monthly income "according & t's po & view' \\
\hline $\begin{array}{l}\text { - Enough for basic needs =adequate } \\
\text { - Not enough for basic needs =inadequate }\end{array}$ & $\begin{array}{c}76 \\
113\end{array}$ & $\begin{array}{l}40.2 \\
59.8\end{array}$ \\
\hline
\end{tabular}


Table 2: Distribution of the studied patients according to their clinical "psychiatric" data $(n=189)$.

\begin{tabular}{|c|c|c|}
\hline Statement & No & $\%$ \\
\hline $\begin{array}{l}\text { Psychiatric diagnosis } \\
\text { - Schizophrenia } \\
\text { - Bipolar affective disorder (BAD) } \\
\text { - Depression } \\
\text { - Obsessive compulsive disorder (OCD) } \\
\text { - Others }\end{array}$ & $\begin{array}{c}91 \\
42 \\
38 \\
8 \\
10\end{array}$ & $\begin{array}{c}48.2 \\
22.2 \\
20.1 \\
4.2 \\
5.3\end{array}$ \\
\hline $\begin{array}{l}\text { Duration of illness } \\
\text { - } 3 \leq 6 \\
\text { - } 6 \leq 9 \\
\text { - } 9 \leq \\
\end{array}$ & $\begin{array}{c}108 \\
40 \\
41 \\
\end{array}$ & $\begin{array}{l}57.2 \\
21.2 \\
21.6\end{array}$ \\
\hline $\begin{array}{l}\text { Type of treatment* } \\
\text { - Pharmacological } \\
\text { - ECT } \\
\text { - Psychotherapy }\end{array}$ & $\begin{array}{c}189 \\
83 \\
41 \\
\end{array}$ & $\begin{array}{l}100 \\
43.9 \\
21.6\end{array}$ \\
\hline $\begin{array}{l}\text { Previous hospitalization } \\
\text { - Yes } \\
\text { - No }\end{array}$ & $\begin{array}{c}175 \\
14\end{array}$ & $\begin{array}{c}92.6 \\
7.4\end{array}$ \\
\hline $\begin{array}{l}\text { If yes number of hospitalizations }(\mathbf{n}=\mathbf{1 7 5}) \\
\text { - Once } \\
\text { - Twice } \\
\text { - Three times } \\
\text { - Four times }\end{array}$ & $\begin{array}{l}65 \\
30 \\
37 \\
43\end{array}$ & $\begin{array}{l}37.1 \\
17.1 \\
21.1 \\
24.7\end{array}$ \\
\hline
\end{tabular}

*Numbers are not mutually exclusive 
Table 3: Mean scores of perceived devaluation/discrimination; internalized stigma of mental illness; and drug attitude among the studied patients $(n=189)$.

\begin{tabular}{|c|c|}
\hline \multicolumn{1}{|c|}{ Variables } & Mean \pm SD \\
\hline Perceived devaluation/discrimination & $3.01 \pm 0.07$ \\
\hline Internalized stigma of mental illness & $2.59 \pm 0.58$ \\
\hline - Alienation (6 items) & $2.75 \pm 0.61$ \\
\hline - Stereotype Endorsement (7 items) & $2.04 \pm 0.54$ \\
\hline - Discrimination Experience (5 items) & $3.16 \pm 0.62$ \\
\hline - Social withdrawal (6 items) & $2.7 \pm 0.92$ \\
\hline - Stigma resistance (5 items) & $1.26 \pm 0.43$ \\
\hline Drug attitude & $1.83 \pm 6.31$ \\
\hline
\end{tabular}

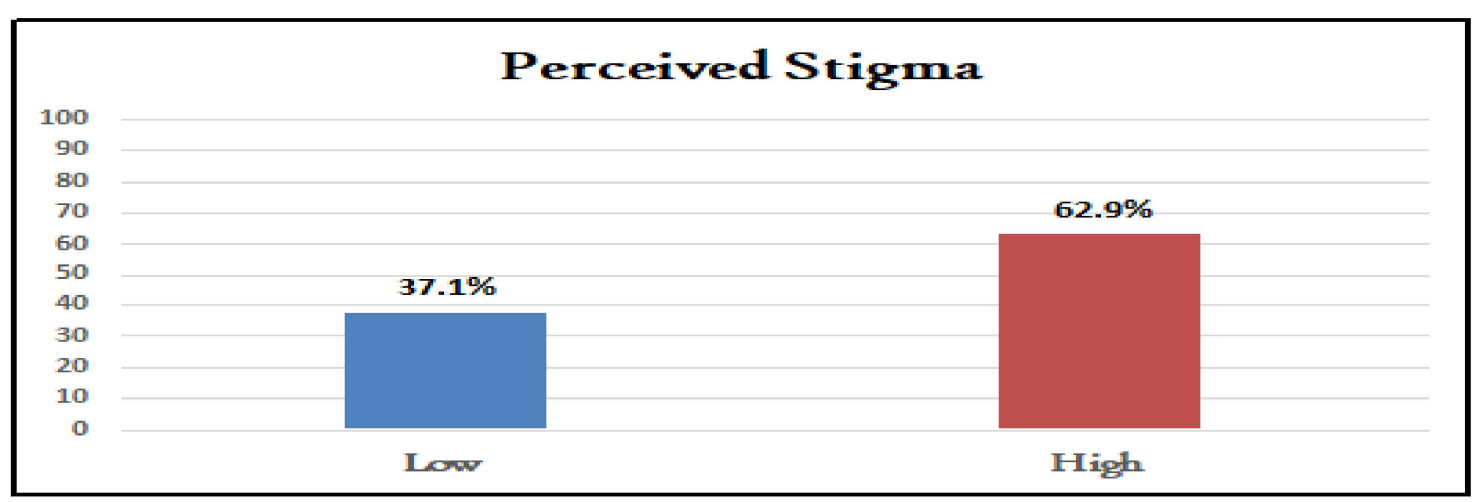

Figure (1): Distribution of the studied patients according to their levels of percieved devaluation/discriminarion (societal stigma) $(n=189)$.

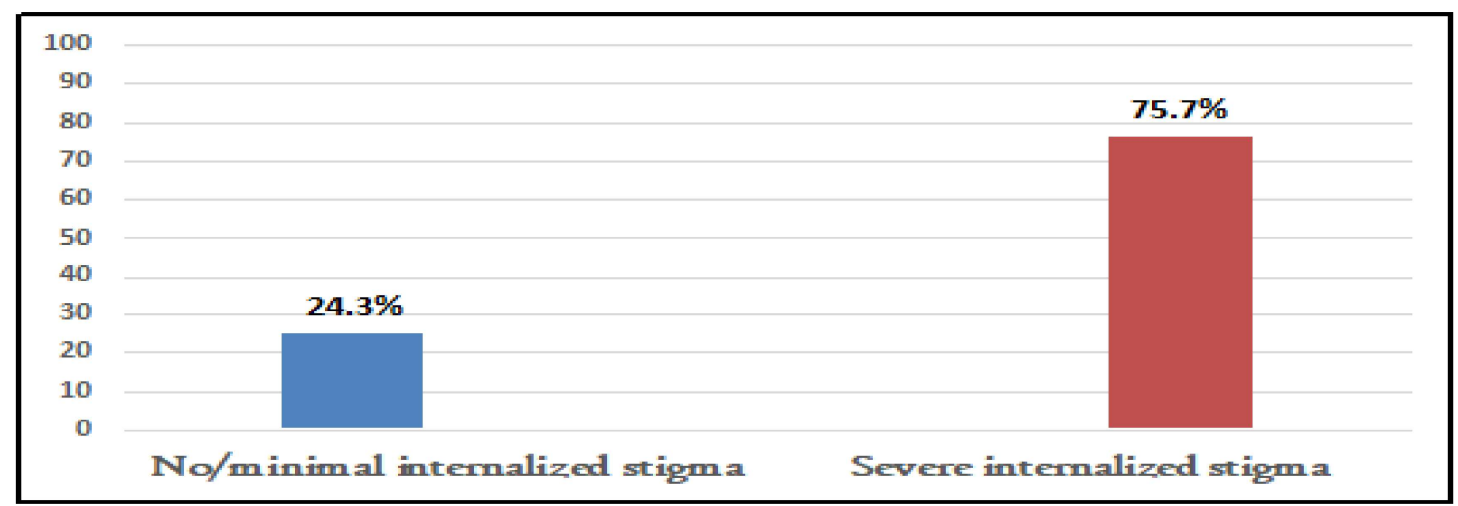

Figure (2): Distribution of the studied patients according to their levels of internalized stigma $(n=189)$. 


\section{Attitude toward PsychotropicMedications}

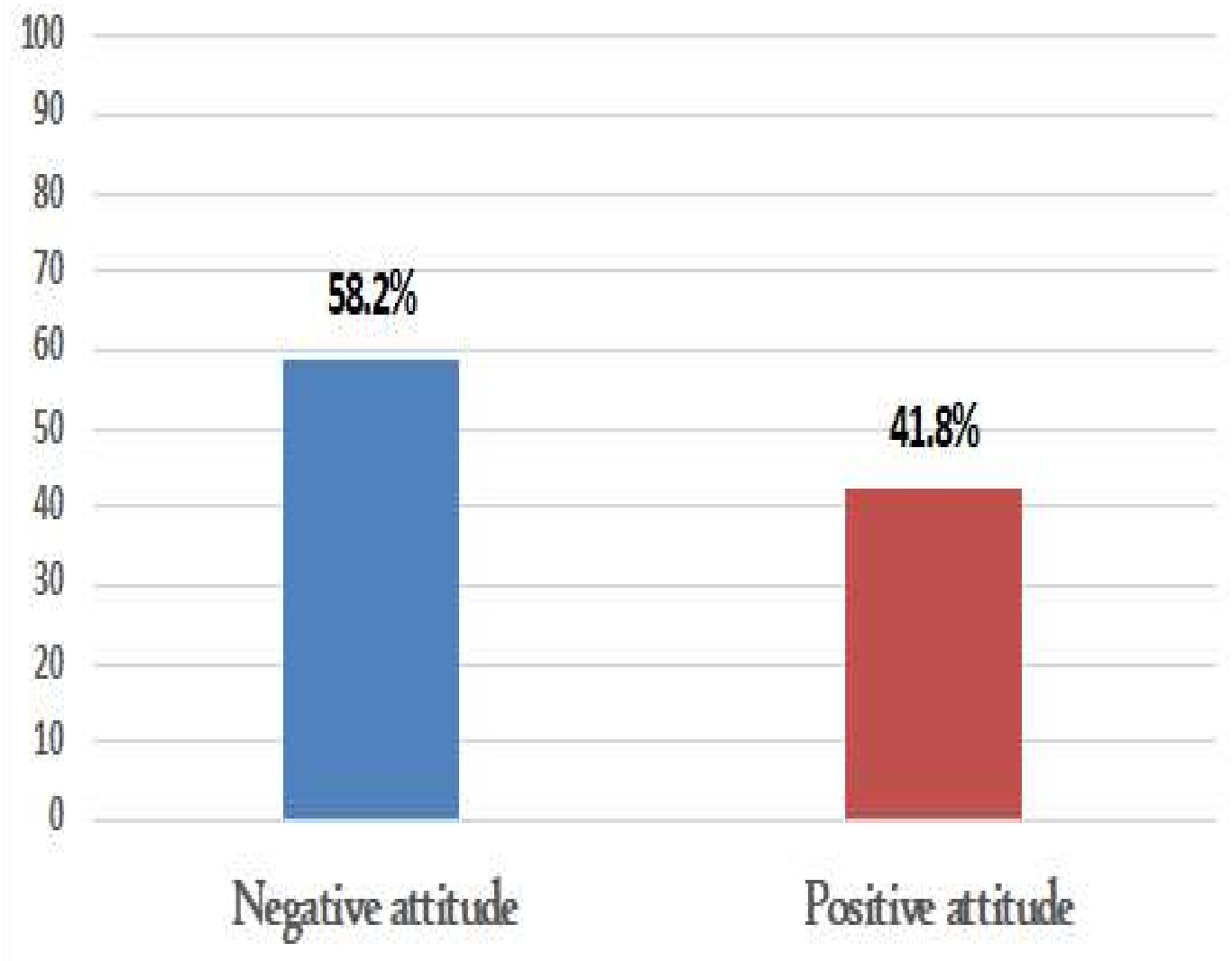

Figure (3): Distribution of the studied patients according to their attitude toward psychotropic medications $(n=189)$. 
Table (4): Relation between demographic characteristics of the studied patients and their perceived devaluation and discrimination, internalized stigma of mental illness, and drug attitude $(n=189)$.

\begin{tabular}{|c|c|c|c|c|c|c|c|c|c|}
\hline \multirow{2}{*}{$\begin{array}{c}\text { Statemen } \\
t\end{array}$} & \multicolumn{2}{|c|}{$\begin{array}{c}\text { perceived } \\
\text { devaluation } \\
\text { and } \\
\text { discrimination } \\
\end{array}$} & \multirow{2}{*}{$\mathbf{X}^{2}$} & \multicolumn{2}{|c|}{$\begin{array}{c}\text { internalized } \\
\text { stigma of mental } \\
\text { illness }\end{array}$} & \multirow{2}{*}{$\mathbf{X}^{2}$} & \multicolumn{2}{|c|}{ drug attitude } & \multirow{2}{*}{$\mathbf{X}^{2}$} \\
\hline & $\begin{array}{l}\text { Low } \\
\text { No. } \\
(\%)\end{array}$ & $\begin{array}{l}\text { High } \\
\text { No. } \\
(\%)\end{array}$ & & $\begin{array}{l}\text { No/mini } \\
\text { mal } \\
\text { No. }(\%)\end{array}$ & $\begin{array}{l}\text { Sever } \\
\text { e } \\
\text { No. } \\
(\%)\end{array}$ & & $\begin{array}{l}\text { Negati } \\
\text { ve } \\
\text { No. } \\
(\%) \\
\end{array}$ & $\begin{array}{l}\text { Positi } \\
\text { ve } \\
\text { No. } \\
(\%)\end{array}$ & \\
\hline \multicolumn{10}{|l|}{ Gender } \\
\hline Male & $\begin{array}{c}33 \\
(30.6)\end{array}$ & $\begin{array}{c}75 \\
(69.4)\end{array}$ & \multirow{2}{*}{1.08} & $13(12.0)$ & $\begin{array}{c}95 \\
(88.0)\end{array}$ & \multirow{2}{*}{$\begin{array}{c}21.7 \\
* *\end{array}$} & $\begin{array}{c}38 \\
(35.1)\end{array}$ & $\begin{array}{l}70 \\
(64.8)\end{array}$ & \multirow[t]{2}{*}{ 5.26* } \\
\hline Female & $\begin{array}{c}27 \\
(33.3)\end{array}$ & $\begin{array}{c}54 \\
(66.7)\end{array}$ & & $\begin{array}{c}31 \\
(38.3) \\
\end{array}$ & $\begin{array}{c}70 \\
(61.7) \\
\end{array}$ & & $\begin{array}{c}42 \\
(51.8) \\
\end{array}$ & $\begin{array}{c}39 \\
(48.2) \\
\end{array}$ & \\
\hline \multicolumn{10}{|l|}{ Age } \\
\hline $18 \leq 28$ & $\begin{array}{c}24 \\
(35.3) \\
\end{array}$ & $\begin{array}{c}44 \\
(64.7) \\
\end{array}$ & \multirow{4}{*}{2.28} & $\begin{array}{c}25 \\
(36.8) \\
\end{array}$ & $\begin{array}{c}43 \\
(63.2) \\
\end{array}$ & \multirow{4}{*}{2.67} & $\begin{array}{c}38 \\
(55.9)\end{array}$ & $\begin{array}{c}30 \\
(44.1) \\
\end{array}$ & \multirow{4}{*}{6.08} \\
\hline $28 \leq 38$ & $\begin{array}{c}35 \\
(35.7)\end{array}$ & $\begin{array}{c}63 \\
(64.3)\end{array}$ & & $\begin{array}{c}12 \\
(12.3)\end{array}$ & $\begin{array}{c}86 \\
(87.7)\end{array}$ & & $\begin{array}{c}63 \\
(64.3)\end{array}$ & $\begin{array}{c}35 \\
(35.7)\end{array}$ & \\
\hline $38 \leq 48$ & $\begin{array}{c}7 \\
(46.7)\end{array}$ & $\begin{array}{c}8 \\
(53.3) \\
\end{array}$ & & $\begin{array}{c}6 \\
(40.0)\end{array}$ & $\begin{array}{c}9 \\
(60.0)\end{array}$ & & $\begin{array}{c}7 \\
(46.7)\end{array}$ & $\begin{array}{c}8 \\
(53.3) \\
\end{array}$ & \\
\hline $\begin{array}{l}\text { More } \\
\text { than } 48\end{array}$ & $\begin{array}{c}4 \\
(50.0)\end{array}$ & $\begin{array}{c}4 \\
(50.0)\end{array}$ & & $\begin{array}{c}3 \\
(37.5)\end{array}$ & $\begin{array}{c}5 \\
(62.5)\end{array}$ & & $\begin{array}{c}2 \\
(25.0)\end{array}$ & $\begin{array}{c}6 \\
(75.0)\end{array}$ & \\
\hline \multicolumn{10}{|c|}{ Educational Status } \\
\hline Illiterate & $\begin{array}{c}8 \\
(27.6)\end{array}$ & $\begin{array}{c}21 \\
(72.4)\end{array}$ & \multirow{5}{*}{3.10} & $3(10.4)$ & $\begin{array}{c}26 \\
(89.6) \\
\end{array}$ & \multirow{5}{*}{3.41} & $\begin{array}{c}20 \\
(69.0) \\
\end{array}$ & $\begin{array}{c}9 \\
(31.0) \\
\end{array}$ & \multirow{5}{*}{5.76} \\
\hline $\begin{array}{l}\text { Primary } \\
e d u .\end{array}$ & $\begin{array}{c}20 \\
(28.6)\end{array}$ & $\begin{array}{c}50 \\
(71.4)\end{array}$ & & $12(17.2)$ & $\begin{array}{c}58 \\
(82.8)\end{array}$ & & $\begin{array}{c}43 \\
(61.4)\end{array}$ & $\begin{array}{c}27 \\
(38.6)\end{array}$ & \\
\hline $\begin{array}{l}\text { Secondary } \\
\text { edu. }\end{array}$ & $\begin{array}{c}5 \\
(17.9)\end{array}$ & $\begin{array}{c}23 \\
(82.1) \\
\end{array}$ & & $3(10.7)$ & $\begin{array}{c}25 \\
(89.7) \\
\end{array}$ & & $\begin{array}{c}18 \\
(64.3) \\
\end{array}$ & $\begin{array}{c}10 \\
(35.7)\end{array}$ & \\
\hline Diploma & $\begin{array}{c}33(62 \\
.3)\end{array}$ & $\begin{array}{c}20(37 \\
.7)\end{array}$ & & $24(45.3)$ & $\begin{array}{c}29 \\
(54.7)\end{array}$ & & $\begin{array}{c}25 \\
(47.2)\end{array}$ & $\begin{array}{c}28 \\
(52.8)\end{array}$ & \\
\hline $\begin{array}{l}\text { Universit } \\
\text { yedu. }\end{array}$ & $\begin{array}{c}4 \\
(44.4)\end{array}$ & $\begin{array}{c}5 \\
(55.6) \\
\end{array}$ & & $4(44.4)$ & $\begin{array}{c}5 \\
(55.6) \\
\end{array}$ & & $\begin{array}{c}4 \\
(44.4)\end{array}$ & $\begin{array}{c}5 \\
(55.6) \\
\end{array}$ & \\
\hline \multicolumn{10}{|c|}{ Occupational Status } \\
\hline $\begin{array}{l}\text { Unemploy } \\
\text { ed }\end{array}$ & $\begin{array}{c}22 \\
(16.4)\end{array}$ & $\begin{array}{c}112 \\
(83.6) \\
\end{array}$ & \multirow{4}{*}{$\begin{array}{l}85.6^{*} \\
*\end{array}$} & $26(19.4)$ & $\begin{array}{c}108 \\
(80.6) \\
\end{array}$ & \multirow{4}{*}{$\begin{array}{c}22.4 \\
* *\end{array}$} & $\begin{array}{c}110 \\
(82.1) \\
\end{array}$ & $\begin{array}{c}24 \\
(17.9) \\
\end{array}$ & \multirow{4}{*}{$\begin{array}{c}108.0 \\
* *\end{array}$} \\
\hline $\begin{array}{l}\text { Private } \\
\text { work }\end{array}$ & $\begin{array}{c}31 \\
(81.6) \\
\end{array}$ & $\begin{array}{c}7 \\
(18.4)\end{array}$ & & $25(65.8)$ & $\begin{array}{c}13 \\
(34.2) \\
\end{array}$ & & 0 & $\begin{array}{c}38 \\
(100)\end{array}$ & \\
\hline $\begin{array}{l}\text { Governme } \\
\text { ntal work }\end{array}$ & $\begin{array}{c}9 \\
(100)\end{array}$ & 0 & & $5(55.6)$ & $\begin{array}{c}4 \\
(44.4) \\
\end{array}$ & & 0 & $\begin{array}{c}9 \\
(100)\end{array}$ & \\
\hline Retired & $\begin{array}{c}8 \\
(100)\end{array}$ & 0 & & $6(75.0)$ & $\begin{array}{c}2 \\
(25.0)\end{array}$ & & 0 & $\begin{array}{c}8 \\
(100)\end{array}$ & \\
\hline
\end{tabular}




\begin{tabular}{|c|c|c|c|c|c|c|c|c|c|}
\hline \multirow{2}{*}{$\begin{array}{c}\text { Statemen } \\
t\end{array}$} & \multicolumn{2}{|c|}{$\begin{array}{c}\text { perceived } \\
\text { devaluation } \\
\text { and } \\
\text { discrimination } \\
\end{array}$} & \multirow{2}{*}{$\mathbf{X}^{2}$} & \multicolumn{2}{|c|}{$\begin{array}{c}\text { internalized } \\
\text { stigma of mental } \\
\text { illness }\end{array}$} & \multirow{2}{*}{$X^{2}$} & \multicolumn{2}{|c|}{ drug attitude } & \multirow{2}{*}{$\mathbf{X}^{2}$} \\
\hline & $\begin{array}{l}\text { Low } \\
\text { No. } \\
(\%)\end{array}$ & $\begin{array}{l}\text { High } \\
\text { No. } \\
(\%)\end{array}$ & & $\begin{array}{l}\text { No/mini } \\
\text { mal } \\
\text { No. }(\%)\end{array}$ & $\begin{array}{c}\text { Sever } \\
\text { e } \\
\text { No. } \\
(\%) \\
\end{array}$ & & $\begin{array}{l}\text { Negati } \\
\text { ve } \\
\text { No. } \\
(\%) \\
\end{array}$ & $\begin{array}{l}\text { Positi } \\
\text { ve } \\
\text { No. } \\
(\%) \\
\end{array}$ & \\
\hline \multicolumn{10}{|l|}{ Residence } \\
\hline Urban & $\begin{array}{c}57 \\
(85.1) \\
\end{array}$ & $\begin{array}{c}10 \\
(14.9)\end{array}$ & \multirow{2}{*}{$\begin{array}{c}102.7 \\
* *\end{array}$} & $33(49.3)$ & $\begin{array}{c}34 \\
(50.7)\end{array}$ & \multirow{2}{*}{$\begin{array}{c}34.9 \\
* *\end{array}$} & $\begin{array}{c}12 \\
(17.9)\end{array}$ & $\begin{array}{c}55 \\
(82.0) \\
\end{array}$ & \multirow{2}{*}{$\begin{array}{c}137.2 \\
* *\end{array}$} \\
\hline Rural & $\begin{array}{c}15 \\
(12.3)\end{array}$ & $\begin{array}{c}107 \\
(87.7) \\
\end{array}$ & & $13(10.7)$ & $\begin{array}{c}109 \\
(89.3) \\
\end{array}$ & & $\begin{array}{c}105 \\
(86.1) \\
\end{array}$ & $\begin{array}{c}17 \\
(13.9)\end{array}$ & \\
\hline \multicolumn{10}{|c|}{ Adequacy of monthly income } \\
\hline Adequate & $\begin{array}{c}31 \\
(40.8)\end{array}$ & $\begin{array}{c}45 \\
(59.2) \\
\end{array}$ & \multirow[b]{2}{*}{0.76} & $36(47.4)$ & $\begin{array}{c}40(52 \\
.6) \\
\end{array}$ & \multirow[b]{2}{*}{$\begin{array}{c}18.6 \\
* *\end{array}$} & $\begin{array}{c}42 \\
(55.3) \\
\end{array}$ & $\begin{array}{c}34 \\
(44.7) \\
\end{array}$ & \multirow{2}{*}{$\begin{array}{c}12.53 \\
* *\end{array}$} \\
\hline $\begin{array}{l}\text { Inadequa } \\
\text { te }\end{array}$ & $\begin{array}{c}39 \\
(34.5)\end{array}$ & $\begin{array}{c}74 \\
(65.5)\end{array}$ & & $15(13.3)$ & $\begin{array}{c}98 \\
(86.7)\end{array}$ & & $\begin{array}{c}65 \\
(57.5)\end{array}$ & $\begin{array}{c}48 \\
(42.7)\end{array}$ & \\
\hline
\end{tabular}

$(*)$ statistically significant at $\mathrm{p}<0.05\left({ }^{* *}\right)$ highly statistically significant at $\mathrm{p}<0.001$. 
Table (5): Relation between medical history of the studied patients and their perceived devaluation and discrimination, internalized stigma of mental illness, and drug attitude $(n=189)$.

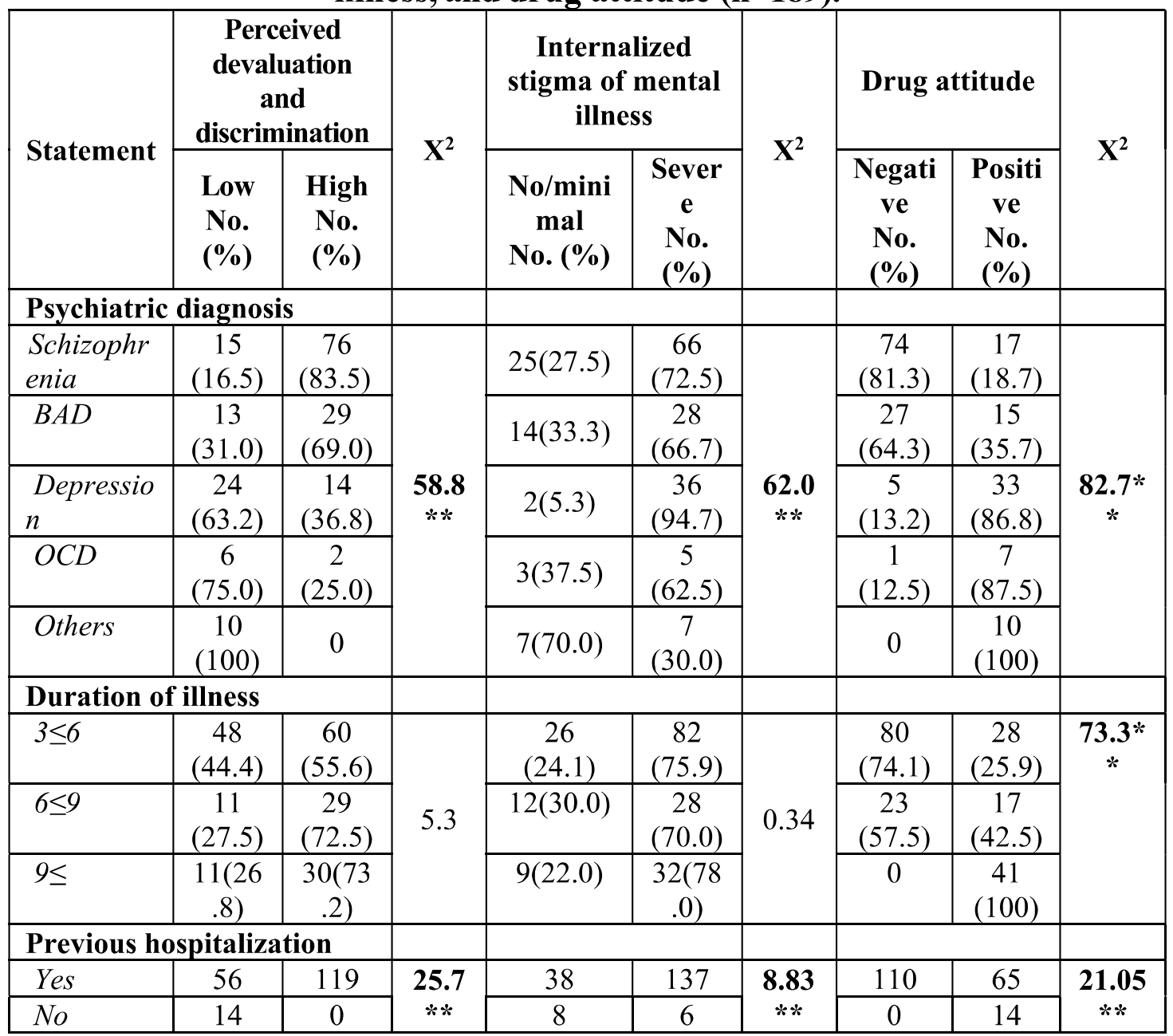

(*) statistically significant at $\mathrm{p}<0.05$

(**)highly statistically significant at $\mathrm{p}<0.001$. 
Table (6): Correlation matrix among perceived devaluation and discrimination, internalized stigma of mental illness, and drug attitude of the studied patients $(\mathbf{n}=189)$.

\begin{tabular}{|l|c|c|}
\hline \multicolumn{1}{|c|}{ Items } & $\begin{array}{c}\text { Internalized } \\
\text { stigma of mental } \\
\text { illness }\end{array}$ & $\begin{array}{c}\text { Perceived } \\
\text { devaluation and } \\
\text { discrimination }\end{array}$ \\
\hline $\begin{array}{l}\text { Perceived devaluation and } \\
\text { discrimination }\end{array}$ & $.739^{* *}$ & \\
\hline Drug attitude & $-.669^{* *}$ & $-.905^{* *}$ \\
\hline
\end{tabular}

$\left.{ }^{*}\right)$ statistically significant at $\mathrm{p}<0.05$

$(* *)$ highly statistically significant at $\mathrm{p}<0.001$.

\section{Discussion}

Stigma affects various aspects of the psychiatric patient's life forcing them to suffer the greatest disease burden without treatment. The current study comprised 189 psychiatric patients with a variety of diagnoses to determine the effect of perceived devaluation, discrimination, and internalized stigma on adherence to medication among patients with mental illness.

Stigma and adherence to psychotropic medication

This study revealed that, two thirds of the studied patients experienced high perceived devaluation/discrimination (societal stigma) as their mean score was 3.01 "above the midpoint of 2.5", three quarters of them experienced severe internalized stigma as the lowest value was found for stigma resistance with a mean score lower than the midpoint of 2.5 (1.26), lower scores on this subscale indicate lower resistance to stigma and thus severe internalized stigma. The other four subscales showed mean scores more than the midpoint, with the highest value for discrimination experience and alienation (3.16 and 2.75 respectively), and less than three fifths of them had negative attitude toward psychotropic medications as their mean score was 2.83. These findings support the indirect significant correlation between patients' attitude towards psychotropic medications in the current study and their levels of internalized (self) stigma, and perceived devaluation/discrimination (societal stigma). Also, the statistically direct significant correlation between internalized (self) stigma and perceived devaluation/discrimination (societal stigma). This result highlights that, the stigma is not confined to negative ideas and stereotypes perceived by society about having a mental illness per se, but also includes patients` feelings of shame, guilt, and other unfavorable attitudes toward mental health treatment, and help- 
seeking, and increases medication discontinuation rates. Although stigma associated with mental illness is a universal phenomenon, its manifestation may differ by cultural attitude. The negative view about mental illness makes people avoid the psychiatric patient and in turn makes the patient avoid treatment. When the patients receive people's judgments and negative stereotypes in the form of avoiding dealing with them, laughing at them, or making fun of them, that enforces them to prefer sorcery and avoid seeking the psychiatrist to avoid being stigmatized as a mental patient.

The finding is congruent with Feldhaus et al., (2018), who investigated the impact of self-stigmatization on medication attitude in schizophrenia patients and mentioned that a higher level of self-stigmatization is related to a more negative attitude towards antipsychotic drug treatment in patients with schizophreniaspectrum disorders.

Also, Kamaradova, Latalova, Prasko, Kubinek, Vrbova, Mainerova, \& Tichackova, (2016), who studied the connection between self-stigma, adherence to treatment, and discontinuation of medication, had mentioned that there was a statistically significant negative correlation between self-stigma and adherence to treatment in all diagnostic groups, as high levels of self-stigma are associated with discontinuation of medications without a psychiatrist's recommendation.

Regarding the direct significant correlation between internalized (self) stigma and perceived devaluation/discrimination (societal stigma) in the present study, Quinn, et al., (2015), explored the relationship between discrimination experiences, anticipated stigma, and self-stigma in an effort to understand how self-stigma might develop, and their finding supports the findings of the present study as their findings suggest that because of prior experiences of discrimination, individuals with mental illness come to expect and anticipate that they will be stigmatized, which, in turn, contribute to believing that the stereotypes involved are true.

Also, Jennings, Cheung, Britt, Goguen, Jeffirs, Peasley, \& Lee, (2015), who examined the role of perceived need for self-reliance in the relationship between perceived societal stigma and self-stigma associated with treatment seeking among college students with mental health problems/concerns, found that perceived stigma affect mental health treatment seeking by increasing individuals' self-stigmatizing attitudes and their preferences for handling mental 
health issues on their own, suggesting that self-reliance may serve as significant barrier to care.

This result is in line with Szcześniak, Kobyłko, Wojciechowska, Klapciński, \&Rymaszewska, (2018), who mentioned that the highest score of ISMI was observed in the alienation domain $(2.63 \pm 0.8)$, while the study population demonstrated a mild level of internalized stigma (2.23 \pm 0.5$)$, and the lowest score was noted in the stereotype endorsement domain (2.08 \pm 0.6$)$.

This result is contradicted with Feldhaus et al., (2018), who mentioned that the highest value of ISMI was found for stigma resistance with a mean score 2.65 thus less internalized stigma. This discrepancy may be related to culture difference and raised public awareness in their study regarding mental illness. Besides, the vast majority of patients had previous experience with psychoeducational interventions and felt sufficiently informed about both their illness and their medication.

Also, Kim, Song, Ryu, Ryu, Kim, Ha, and Cho, (2015), mentioned that the mean ISMI score per item in their study was relatively low (2.06 points, which was 49.40 points divided by 24 items), and the percentage of patients who reported a high level of internalized stigma (above the midpoint of the ISMI was also lower $(8.1 \%)$. This difference might be caused by the composition of the subjects in their study or the location of data collection.

Demographic factors and the study variables (stigma and medication adherence)

Regarding demographic data of the participants, data analysis shows that half of them were in the age group from 28 to less than 38 years old, although there was no significant relation between participants` age and perceived societal stigma, internalized stigma, or drug attitude, the middle age group shows a positive attitude toward medication. It is possible that this age group might have a more in-depth and targeted view of many situations, as well as being more experienced and accepted. That was supported with Gaber \& Wadie, (2016), who studied the perceived self-stigma, adherence to treatment and discontinuation of medication among schizophrenic patients and occluded that, more than half of the studied participants aged between 26 to 35 years old with no significant association to perceived self-stigma, discontinuation of medication and adherence to medication. This result is also in line with Yen, Chen, Lee, Tang, Yen, \& Ko, (2005), who studied self-stigma and its correlates among 
outpatients with depressive disorders and they didn't find any statistically significant relation between stigma and sex or age.

The current study revealed that more than half of the studied patients were male. Also, there were statistically significant differences between gender of the studied patients and levels of internalized stigma of mental illness, and patient attitude toward psychotropic medication, as male gender was a predictor of increased self-stigmatization as most of the studied male patients had severe internalized stigma for mental illness while nearly three quarters of them had positive drug attitudes concerning psychotropic medications. These results can be interpreted as mental disorder and its related impairments are perceived as a sign of weakness and inadequacy by the society, putting more emphasis on masculine schemata, and as being incongruous with the expected gender role, especially in our eastern societies. As a result, those patients are facing a serious threat to their "male identity." This result could also be interpreted as male patients have high follow rate at outpatient clinics to avoid symptom persistence and/or progression, social dysfunction (dismissed from their employment), and additional negative consequences, all of which could reinforce self-stigmatization and related negative emotional reactions.

This result is contradicted with Asrat, B., Ayenalem, A. E., \& Yimer, T. (2018), who mentioned that the prevalence of internalized stigma was $32.1 \%$ among people living with mental illness in Dilla University Referral Hospital. Being female, medication nonadherence, and lack of family support were factors independently associated with internalized stigma. Also, Khan, Kausar, Khalid, \& Farooq, (2015), mentioned that both men and women experienced considerably high level of associated stigma and discrimination due to their mental illness. However, women in comparison to men experience significantly greater level of internalized stigma especially in domains of discrimination experience and social withdrawal. This is also in contrast with Brohan, Elgie, Sartorius, Thornicroft (2010), who found higher levels of self-stigmatization in females with schizophrenia.

Regarding the level of education and work status of the studied participants. The current study pointed out that despite the majority of them being educated and nearly half of them receiving secondary and diploma education, they were stigmatized and half of them were less adhered to medication, with no statically significant relation between educational level and the study variables. These results were supported by the absence of statistically significant differences between the subgroups. 
The current study also highlights a statically significant relation between work status and studied variables, as slightly more than two thirds of the studied participants were unemployed, the majority of them showed severe perceived societal and self-sigma and negative drug attitude. In contrast nearly one third who had private work showed minimal perceived societal and self-sigma and positive drug attitude. This study result is supported by Kamaradova et al., (2016), who revealed that self-stigma is negatively correlated with work status.

Concerning the monthly income more than half of studied participant claimed that the monthly income was inadequate, this result can be linked to the previous mentioned result " slightly more than two thirds of the studied participants were unemployed" and relay on their families in addition monthly income and financial burden is related to perceived societal, self-stigma and negative drug attitude.

\section{Clinical data "psychiatric history" and the study variables (stigma and medication adherence)}

Regarding the clinical "psychiatric" data of the studied patients, the present study showed that less than half of them were diagnosed with schizophrenia, and more than two fifths of them were diagnosed with mood disorders, also there were statistical significant relations between psychiatric diagnosis and the studied variables, as patients with schizophrenia and mood disorder experienced high perceived devaluation/discrimination (except for patients with depression-as the nature of depression includes introjection of negative thoughts and guilt based on the presence of thought distortion, as they perceive the problems as being their fault), severe internalized (self) stigma and negative attitude toward psychotropic medication.

This result is in line with Szcześniak, et al., (2018), who stated that, the highest degree of internalized stigma was present in participants with unipolar affective disorder and was of moderate severity (2.46 \pm 0.6$)$, while the level was moderate in the alienation domain $(2.85 \pm 0.8)$.

This result is contradicted with Kamaradova et al., (2016), who stated that there were no statistically significant differences in the mean ISMI scores between individual diagnostic categories.In addition, the duration of mental illness is linked to drug attitudes. More than half of the studied participants have suffered from a psychiatric disorder for 3 up to 6 years. Three quarters of them reported severe internalized self-stigma and a negative drug attitude. Meanwhile, 
participants have suffered from psychiatric disorders for a prolonged duration (more than 9 years) showed more positive drug attitude despite of feelings of discrimination, devaluation and internalized stigma. The previous result may be due to the willingness of the patient with a prolonged duration of illness to maintain functioning and minimize the negative consequences of their illness, while the mentally ill patient with a short duration of illness faces difficulties handling the disease impact and consequence impairment in function that cause feelings of discrimination and abandonment.

Gaber \& Wadie, (2016), supported the present study results as they pointed out that there was a positive relation between duration and onset of the disease and experiencing self-stigma and drug attitude.

Further findings of the present study indicated the significant relation between previous history of hospitalization and perceived societal stigma, internalized stigma and drug attitude, as almost all of the studied participants had a history of previous hospitalization associated with high perceived and severe internalized stigma and negative drug attitude. More than one third of them were hospitalized once and slightly half of them were hospitalized 3 to 4 times. This result magnified the impact of frequent hospitalization on increasing feelings of discrimination, stereotype, and inadequacy, which leads to more deterioration in patients' level of functioning, discontinuation of medication, and recurrent admission. This result is in line with Kamaradova et al., (2016), who stated that self-stigma also negatively correlated with education, and positively with a number of hospitalizations and number of psychiatrists visited.This result is in contradicted with Szcześniak, et al., (2018), who mentioned that the number of hospital admissions was not significantly correlated with internalized stigma.

Conclusion: The present study results concluded that, the levels of societal stigma and internalized (self) stigma were determined to be high and negatively affected the patients' attitudes toward adherence to psychotropic medications. Psychiatric diagnosis and duration of illness were factors affecting internalized stigma, societal stigma, and drug attitude. Also, increased duration of illness was contributed to positive drug attitude; and societal and internalized stigma were contributed to readmission to hospital. 


\section{Recommendations}

1. A psycho-educational social skill training sessions should be implemented as soon as possible in outpatient clinics to develop and prompt mentally ill patients' adherence to treatment.

2. Routine stigma assessment in daily clinical practice to be followed by stigma intervention programs

3. Families should be included in educational or supportive counseling session to develop and improve their caring abilities for mentally ill patient.

4. Develop a public health awareness program to raise the orientation toward the nature of psychiatric disorders and alleviate stigma of seeking treatment.

\section{Reference}

Asrat, B., Ayenalem, A. E., \& Yimer, T. (2018). Internalized stigma among patients with mental illness attending psychiatric follow-up at Dilla University Referral Hospital, Southern Ethiopia. Psychiatry journal, 2018.

Awad, A. G. (1993). Subjective response to neuroleptics in schizophrenia. Schizophrenia bulletin, 19(3), 609-618.

Boyd, J. E., Adler, E. P., Otilingam, P. G., \& Peters, T. (2014). Internalized Stigma of Mental Illness (ISMI) scale: a multinational review. Comprehensive Psychiatry, 55(1), 221-231.

Brohan, E., Elgie, R., Sartorius, N., Thornicroft, G., (2010). Self-stigma, empowerment andperceived discrimination among people with schizophrenia in 14 European countries: the GAMIAN-Europe study. Schizophr. Res. 122, $232-$ 238. http://dx.doi.org/10. 1016/j.schres.2010.02.1065.

Corrigan, P. W., \& Rao, D. (2012). On the self-stigma of mental illness: Stages, disclosure, and strategies for change. The Canadian Journal of Psychiatry, 57(8), 464-469.

Corrigan, P. W., Rafacz, J., \& Rüsch, N. (2011). Examining a progressive model of self-stigma and its impact on people with serious mental illness. Psychiatry research, 189(3), 339-343.

Demoz, Z., Legesse, B., Teklay, G., Demeke, B., Eyob, T., Shewamene, Z., \& Abera, M. (2014). Medication adherence and its determinants among psychiatric patients in an Ethiopian referral hospital. Patient preference and adherence, 8, 1329. 
Feldhaus, T., Falke, S., von Gruchalla, L., Maisch, B., Uhlmann, C., Bock, E., \& Lencer, R. (2018). The impact of self-stigmatization on medication attitude in schizophrenia patients. Psychiatry research, 261, 391-399.

Gaber, N., \& Wadie, N. (2016). Perceived Self-Stigma, Adherence to Treatment and Discontinuation of Medication among Schizophrenic Patients. World Journal of Nursing Sciences, 2(3), 61-70.

Jenkins, J. H., \& Carpenter-Song, E. A. (2009). Awareness of stigma among persons with schizophrenia: marking the contexts of lived experience. The Journal of nervous and mental disease, 197(7), 520-529.

Jennings, K. S., Cheung, J. H., Britt, T. W., Goguen, K. N., Jeffirs, S. M., Peasley, A. L., \& Lee, A. C. (2015). How are perceived stigma, self-stigma, and selfreliance related to treatment-seeking? A three-path model. Psychiatric Rehabilitation Journal, 38(2), 109.

Kamaradova, D., Latalova, K., Prasko, J., Kubinek, R., Vrbova, K., Mainerova, B., \& Tichackova, A. (2016). Connection between self-stigma, adherence to treatment, and discontinuation of medication. Patient preference and adherence, 10,1289 .

Keen, N., George, D., Scragg, P., \& Peters, E. (2017). The role of shame in people with a diagnosis of schizophrenia. British Journal of Clinical Psychology, 56(2), 115-129.

Khan, N., Kausar, R., Khalid, A., \& Farooq, A. (2015). Gender differences among discrimination \& stigma experienced by depressive patients in Pakistan. Pakistan journal of medical sciences, 31(6), 1432.

Kim, W. J., Song, Y. J., Ryu, H. S., Ryu, V., Kim, J. M., Ha, R. Y., \& Cho, H. S. (2015). Internalized stigma and its psychosocial correlates in Korean patients with serious mental illness. Psychiatry research, 225(3), 433-439.

Lee, J. M. (2016). Diminishing Stigma Sentiments in Individuals with Depression: Sociopsychological Predictors of Deflecting and Challenging Coping Orientations (Doctoral dissertation, Walden University).

Link, B. G., Yang, L. H., Phelan, J. C., \& Collins, P. Y. (2004). Measuring mental illness stigma. Schizophrenia bulletin, 30(3), 511-541.

Quinn, D. M., Williams, M. K., \& Weisz, B. M. (2015). From discrimination to internalized mental illness stigma: The mediating roles of anticipated discrimination and anticipated stigma. Psychiatric Rehabilitation Journal, 38(2), 103. 
Ritsher, J. B., Otilingam, P. G., \&Grajales, M. (2003). Internalized stigma of mental illness: psychometric properties of a new measure. Psychiatry research, 121(1), 31-49.

Sarısoy, G., Kaçar, Ö. F., Pazvantoğlu, O., Korkmaz, I. Z., Öztürk, A., Akkaya, D., \& Sahin, A. R. (2013). Internalized stigma and intimate relations in bipolar and schizophrenic patients: a comparative study. Comprehensive psychiatry, 54(6), 665-672.

Sorsdahl, K. R., Kakuma, R., Wilson, Z., \& Stein, D. J. (2012). The internalized stigma experienced by members of a mental health advocacy group in South Africa. International Journal of Social Psychiatry, 58(1), 55-61.

Szcześniak, D., Kobyłko, A., Wojciechowska, I., Klapciński, M., \&Rymaszewska, J. (2018). Internalized stigma and its correlates among patients with severe mental illness. Neuropsychiatric disease and treatment, 14, 2599.

Tanabe, Y., Hayashi, K., \&Ideno, Y. (2016). The Internalized Stigma of Mental Illness (ISMI) scale: validation of the Japanese version. BMC psychiatry, 16(1), 1-8.

Teferra, S., Hanlon, C., Beyero, T., Jacobsson, L., \& Shibre, T. (2013). Perspectives on reasons for non-adherence to medication in persons with schizophrenia in Ethiopia: a qualitative study of patients, caregivers and health workers. $B M C$ psychiatry, 13(1), 1-9.

Yen, C. F., Chen, C. C., Lee, Y., Tang, T. C., Yen, J. Y., \& Ko, C. H. (2005). Selfstigma and its correlates among outpatients with depressive disorders. Psychiatric Services, 56(5), 599-601.

Yılmaz, E., \&Okanl, A. (2015). The effect of internalized stigma on the adherence to treatment in patients with schizophrenia. Archives of Psychiatric Nursing, 29(5), 297-301.

Zäske, H., Degner, D., Jockers-Scherübl, M., Klingberg, S., Klosterkötter, J., Maier, W., \& Gaebel, W. (2016). Experiences of stigma and discrimination in patients with first-episode schizophrenia. Der Nervenarzt, 87(1), 82-87.

Krejcie, R. V., \& Morgan, D. W. (1970). Determining sample size for research activities. Educational and psychological measurement, 30(3), 607-610.

Ritsher, J. B., \& Phelan, J. C. (2004). Internalized stigma predicts erosion of morale among psychiatric outpatients. Psychiatry research, 129(3), 257-265. 


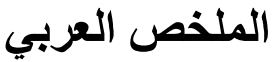

\section{تأثير إنخفاض القيمة المدركة والتمييز، والوصم الأتى على الالتزام بالدواء بين مرضى الألى

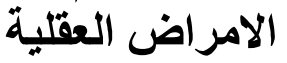

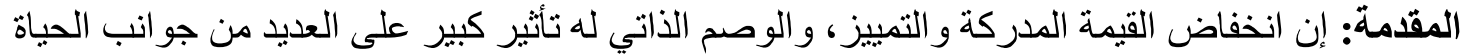

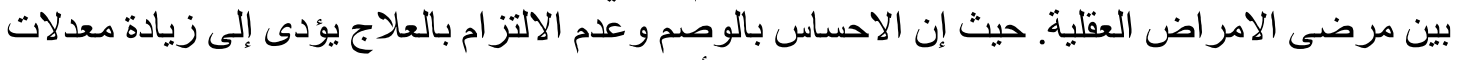
الحجز بالمؤسسات العلاجية وتطور المرض العنف وتفاقم الأعر اض.

الهاف: أجريت هذه الدراسة لتحديد تأثير انخفاض القيمة الددركة و التمييز والوصم الذاتي على الالتزام بالدو اء بين مرضى الامر اض العقلية.

طريقة البحث: تم استخدام التصميم الوصفي الارتباطي في الدراسة الحالية لعينة مكونة من 189 مريضًا

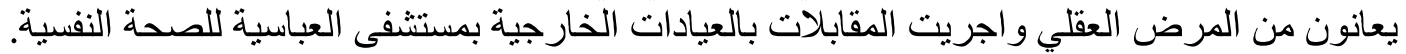

أدوات البحث: تم استخدام أربع أدوات لجمع البيانات تشمل 1. البيانات الديمو غر افية والطبية، و 2.

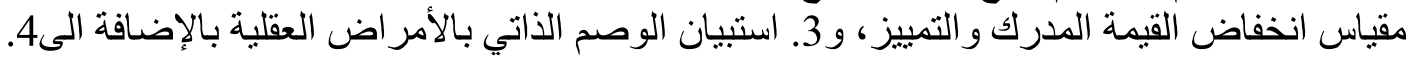

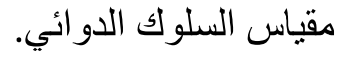

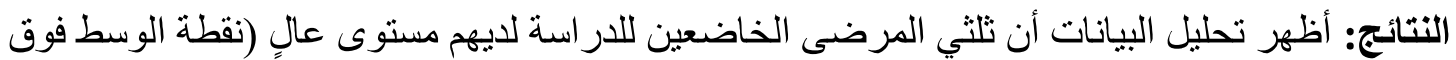

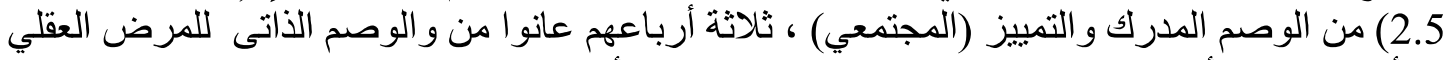

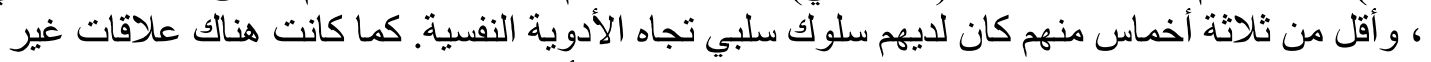

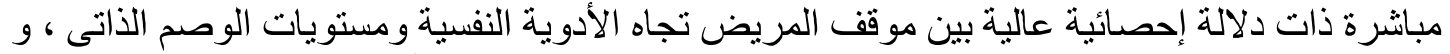

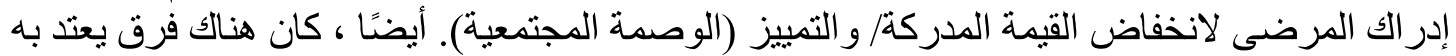

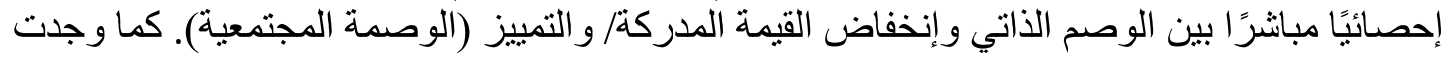

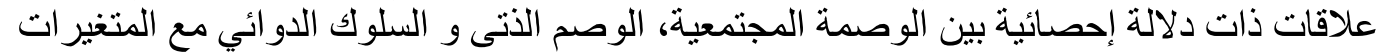

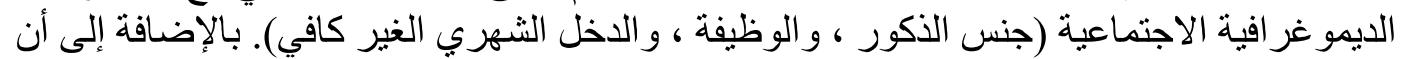

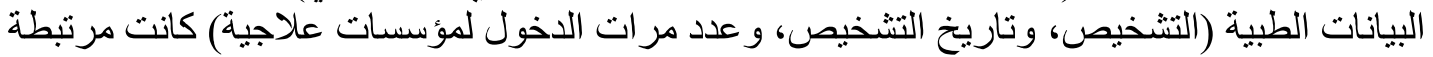
بمتغيرات الدراسة: الوصمة المجتمعية الدركة، ومشاعر الوصم الذاتى ،و السلوك الدوات الدئي.

الخلاصة: تم تحديد مستويات عالية من إدر الك المرضى للوصمة المجتمعية ووصم الذات و التي تؤثر سلبًا على سلوك المرضى تجاه الالتز ام بالأدوية النفسية .

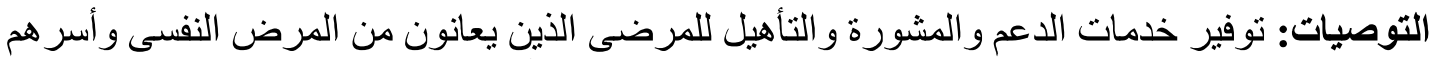
لتحسين مقاومتهم للوصم المجتمعى و الذاتى ، وتعزيز الالتنز ام بالأدوية النفسية. 